duktion der Radiojod-Aufnahme sowie auf Knochenmetastasen. Die offene monozentrische prospektive Phase-II-Studie über 26 Wochen mit anschließender Verlängerungsphase umfasste 31 Patienten mit progredientem metastasiertem oder lokal fortgeschrittenem Radiojod-refraktärem differenziertem Schilddrüsenkarzinom.

Nach 26 Wochen unter Sorafenib war in der Ganzkörperszintigrafie keine Reinduktion der Radiojod-Aufnahme im Bereich der Metastasen nachweisbar. Dennoch profitierten 19 Patienten (59\%) klinisch von dem Multi-Kinase-Hemmer:
Acht $(25 \%)$ zeigten ein partielles Ansprechen und elf $(34 \%)$ eine stabile Erkrankung, sieben (22\%) eine Progression. Bei Patienten mit Knochenmetastasen erwies sich Sorafenib als signifikant weniger effektiv, obwohl $22 \%$ dieser Patienten einen klinischen Nutzen hatten (9\% partielles Ansprechen, 13\% stabile Erkrankung). Insgesamt lag das geschätzte mediane progressionsfreie Überleben bei 58 Wochen. Die Therapie war allerdings mit erheblichen Nebenwirkungen verbunden.

Fazit: Bei Patienten mit metastasiertem differenziertem Schilddrüsenkarzinom lassen sich mit Sorafenib günstige Effekte erzielen. Knochenmetastasen schmälern jedoch die Erfolgsaussichten. Im Ganzkörper-Szintigramm ist keine Reinduktion der Radiojod-Aufnahme nachweisbar.

uwe

Hoftijzer $\mathrm{H}$ et al. Beneficial effects of sorafenib on tumor progression, but not on radioiodine uptake, in patients with differentiated thyroid carcinoma.

Eur J Endocrinol 2009; 161: 923-31.

\title{
Lebensqualität nach Nasenchirurgie nicht immer besser
}

\author{
Wer sich vor einer Nasen-Operation einer wenig beeinträchtigten Lebensqualität erfreuen \\ kann, profitiert entsprechend kaum von dem Eingriff. Das gilt vor allem für Patienten, die \\ sich einer Septumoperation unterzogen werden.
}

$\mathrm{D}$ arauf weist eine prospektive Studie aus Dresden und Dortmund mit 788 Patienten (davon 492 Männern) im Alter zwischen 9 und 81 Jahren hin. 336 von ihnen wurden endoskopisch an den Nasennebenhöhlen operiert, 358 am Septum, bei 94 erfolgte eine Sinus-Op. zusammen mit einer Septumkorrektur. Die Lebensqualität vor der Operation ermittelten die Untersucher mit dem standardisierten SF-36-Fragebogen (Short Form Health Survey). Außerdem erfassten sie die subjektiven sinonasalen Symptome mit dem Rhinosinusitis Disability Index (RSBI). 29,5\% der Patienten beurteilten ihre Beschwerden vor der Operation als schwerwiegend, $61,2 \%$ als mäßiggradig und $9,3 \%$ als gering. Eine im Vergleich zur Allgemeinbevölkerung beeinträchtigte Lebensqualität fand sich vor allem bei den Patienten mit Nasennebenhöhleneingriffen, dagegen kaum bei Septumpatienten.

Von 361 Patienten standen Angaben zu Lebensqualität und Symptomschwere auch für einen Zeitpunkt von im Median 4 Monaten nach dem Eingriff zur Verfügung. Zu diesem Zeitpunkt beurteilten 2,8\% der Patienten ihre Symptome immer noch als schwerwiegend, $35 \%$ als mäßig stark und $62,2 \%$ als ge- ring. Subjektiv hatte sich die Symptomatik damit bei mehr als $80 \%$ der Patienten gebessert. Die Lebensqualität stieg nach Sinusoperation häufig ebenfalls deutlich an. Da sie bei Septumpatienten im Mittel zuvor kaum beeinträchtigt war, blieb bei diesen Patienten ein klarer Benefit der Nasenchirurgie für die Lebensqualität aus.

Fazit: Die funktionelle endoskopische Nasenchirurgie kann subjektive sinonasale Symptome effektiv verbessern. Eine bessere Lebensqualität resultierte daraus aber nur für die Patienten, die sich vorher diesbezüglich auch deutlich beeinträchtigt gefühlt haben, und das waren in dieser Erhebung nur diejenigen mit Nebenhöhlenbeschwerden. Langzeituntersuchungen sollen nun zeigen, ob der günstige Effekt der Sinusoperationen auf die Lebensqualität auch länger anhält.

Croy l et al. Quality of life following nasal surgery. Laryngoscope 2010; 120: 826-31.

\section{Kommentar}

Untersuchungen zur Verbesserung der Lebensqualität nach Nasenchirurgie bei Patienten mit chronischer Rhinosinusitis und behinderter Nasenatmung liegen schon seit Längerem vor. Die Ergebnisse der bisherigen Studien haben in der Regel zeigen können, dass sie sich postoperativ verbessert.

Umso mehr verwundern die Daten der vorliegenden Arbeit - und zwar nicht nur den Leser und Rezensenten, sondern offenbar auch die Autoren selber - nach denen die Patienten, die ausschliesslich am Septum operiert wurden, auch postoperativ weiter über eine Einschränkung ihrer Lebensqualität klagten.

Die Autoren führen als mögliche Ursache den Zeitraum von 4 !! Monaten bis zur postoperativen Kontrollbefragung an, den sie für nicht ausreichend halten. Dieser Einschätzung können wir uns nicht anschließen, 4 Monate nach Septum-OP sollte der Heilungsverlauf lange abgeschlossen und die Funktion signifikant besser sein; ist dem nicht so, muss es an der Indikationsstellung und/oder der Op-Technik liegen.

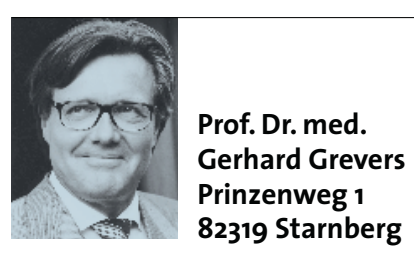

